\title{
Discussion of "The Ketamine Model of the Near-Death Experience: A Central Role for the N-Methyl-D-Aspartate Receptor," by Karl L. R. Jansen
}

\author{
Stuart W. Twemlow, M.D.
}

Topeka Institute for Psychoanalysis, Topeka, KS

University of Kansas School of Medicine, Wichita, KS

\author{
Glen O. Gabbard, M.D. \\ The Menninger Foundation, Topeka, KS \\ Topeka Institute for Psychoanalysis, Topeka, KS
}

ABSTRACT. We review strengths and weaknesses of Karl Jansen's approach to the near-death experience (NDE). Strengths include his limited goals and avoidance of the trap of explaining all features of the NDE with his theory, although he surprisingly misunderstood our previously published position. Additionally, we applaud the possible intersection of psychological and biological theories, demonstrated in Jansen's biochemical explanations for the individualized variations in manifestation and adaptive role of the NDE. However, he failed to take into account the pitfalls in the use of analogy, modeling oversimplification, and in taking association as causality and causes as meaningful, in the arguments for his theory.

Stuart W. Twemlow, M.D., is in the private practice of psychiatry and psychoanalysis; serves on the Faculty of the Topeka Institute for Psychoanalysis, Menninger Foundation, Topeka, KS, and as Clinical Professor of Psychiatry at the University of Kansas School of Medicine, Wichita, KS; and is Chief Instructor at the School of Martial and Meditative Arts in Topeka, KS. Glen O. Gabbard, M.D., is Director and Training and Supervising Analyst at the Topeka Institute for Psychoanalysis, and the Bessie Callaway Distinguished Professor at the Karl Menninger School of Psychiatry and Mental Health Sciences. Reprint requests should be addressed to Dr. Twemlow at 5040 S.W. 28th Street, Topeka, KS 66614. 
We welcome the opportunity to discuss Karl Jansen's paper, which is one more contribution to the growing literature that attempts to explain subjective experience through the use of neurochemical models. Just as previous models of psychosis have used amphetamine and lysergic acid diethylamide (LSD) as the basis of chemical analogues, Jansen has chosen to use the anesthetic ketamine as a model for the near-death experience (NDE). In so doing, he attempted to link physical conditions that have been temporally associated as precipitants of NDEs, particularly hypoxia and related blood gas abnormalities, ischemia, hypoglycemia, and temporal lobe epilepsy.

We certainly do not need to remind him that temporal association with a variety of different physical and psychological precipitants does not prove a causal relationship. In the same vein, a chemically analogous state does not in any way provide conclusive evidence for the causation of a naturally occurring psychological phenomenon such as the near-death experience. We do not intend to review the logical arguments concerning problems inherent in such models except to point out that analogical reasoning, although tempting to invoke, is fraught with interpretive difficulties when not acknowledged as such.

In general, we find that Jansen's hypothesis has considerable appeal and is deserving of serious study. He wisely eschewed any effort to explain all NDEs based on the glutamate hypothesis. Indeed, he ended up reaching the same conclusion that we did more than a decade ago (Gabbard and Twemlow, 1984) that the NDE is probably the final common expression of several different causes. Indeed, we see a possible intersection between our own psychoanalytic perspective and Jansen's neurochemical model.

As we have noted previously (Gabbard and Twemlow, 1991), often people who think they are near death when they are not even in serious jeopardy may nevertheless have a near-death experience. We suggested at the time that the thought of death as well as the physical threat of death can precipitate an elaborate psychological defense mechanism activated by this perception, however misguided it may be. This defensive effort has as its goal the eradication of anxiety and the replacement of that anxiety with a soothing state of mind. In keeping with the principles of overdetermination and multiple causation, the precipitant can be conscious or unconscious in nature.

Moreover, research on near-death experiences in children (Gabbard and Twemlow, 1984) has demonstrated that young children who have no concept of death are nevertheless capable of having near-death 
experiences. Death is omnipresent in the human psyche, however, and although an infant may have no concept of death, it certainly has a sense of dread. In other words, having emerged from the pain of birth itself, the baby screaming with hunger and demanding to be fed cannot name the catastrophe that is feared, even though a terrible threat is experienced. This primitive dread is what Wilfred Bion referred to as catastrophe (Bion, 1963). We postulated that all of us live with the unconscious awareness that a disaster from out of the blue may snuff us out at any moment and that this reality never entirely disappears from our thinking.

In Bion's view, faith is what enables us to get from one moment of catastrophe to another while maintaining some semblance of sanity. The imagery of the near-death experience reflects this faith in that a catastrophe is transformed into a beautiful transcendent event. In speculating about the origins of the adaptive defense mechanism of the near-death experience, we considered the contribution of genetic factors, and it is possible that Jansen's hypothesis may help to clarify some of the biochemical underpinnings of the psychological defense. Specifically, the full-blown NDE might be a subjective response to neuroprotective chemicals that prevent the toxic effect of glutamate flooding. Given the ubiquity of the threat of death, no great leap of imagination is required to speculate that there could be considerable survival value in such a feature within the human genome.

In a previous paper, we concluded, "The dread of oblivion is not an issue that rears its ugly head only when one's survival is literally threatened. It is background noise that haunts us day and night as we frantically strive to deny our own mortality through a myriad of self-deceptions" (Gabbard and Twemlow, 1991, p. 46). Jansen may have found a biological metaphor for the way in which the human brain adapts itself to the sometimes extreme conscious and unconscious stresses of life that we view psychologically as defensive processes.

Having applauded the creativity of his thinking, we would now like to turn our attention to what we view as some limitations of Jansen's model. Central to his perspective was the following line of reasoning: Some of the conditions temporally associated with NDEs release a flood of the chemical glutamate, which can, if unmodified, result in death of neurons. This neurotoxicity is blocked by ketamine, resulting in a dissociative NDE-like state. He then postulated that in certain individuals who are not under the influence of ketamine, 
particular substances may bind protectively to the same receptors to prevent toxicity to neurons. Thus the theory implied a unique mechanism for release of as yet unidentified brain chemicals that block the neurotoxic effect of glutamate. Jansen noted that this hypothetical defensive flood of substances to prevent cell death was the only speculative aspect of his model. Be that as it may, his entire model pivoted on this highly speculative point! Because the paper was replete with impressive scholarship, a casual reader may come away with the impression that the model is a great deal less speculative than it actually is.

Also, at times Jansen glossed over highly controversial issues with deceiving oversimplification. Consider, for example, his statement that "there is overwhelming evidence from thousands of studies relating brain events to alterations in mental state that 'mind' results from neuronal activity" (p. 6). In this rather glib statement Jansen has reduced the complex evidential and philosophical issues involved in the mind/body problem to a rather simple matter: What we know as mind is the result of neuronal activity. This view does not address rigorous critiques of this idea, such as that of John Searle (1992), who made a persuasive case that while states of consciousness may be properties of the brain, they certainly are not reducible to neural activity. The essence of "mind" in Searle's view is consciousness. He noted that materialist perspectives on the concept of "mind" systematically omit conscious experience either by identifying it with something else that is not directly related to consciousness or by leaving it out entirely. There is a fundamental difference between conscious phenomena and neurophysiological phenomena that makes it impossible to reduce consciousness to neuronal activity alone. Consciousness can never be described from an "objective" third-person perspective and is therefore ontologically subjective. Searle also eschewed Cartesian dualism, and he suggested that the irreducible nature of conscious states does not imply a dualistic view. He emphatically stated that materialism and dualism are not the only choices to deal with the mind/body problem. One does not need to choose between them, and the polarization between dualistic and materialistic assumptions is archaic, in Searle's view.

Blurring this distinction between the realm of the psychological and the realm of the biological created further difficulties in articulating Jansen's model. A thought can act as a stressor for the glutamate flood, just as dramatically as any external or physiological event. Researchers studying posttraumatic stress disorder (PTSD) 
have repeatedly observed that a cognition related to the meaning of a trauma can precipitate the symptoms of posttraumatic stress disorder, which obviously have neurophysiological correlates. Those flashbacks produced in PTSD are characterized by richness, complexity, and idiosyncratic meanings that are not fully reducible to chemical explanations.

Jansen has failed to distinguish clearly between causes and meanings. Neurochemical phenomena can be interpreted in a variety of ways, depending on the meanings attributed to them by a particular individual's unique psychological features. The examples used by Jansen illustrate this point compellingly. He quoted Stanislav Grof, for example, as saying, "If you have a full-blown experience of ketamine, you can never believe there is death or that death can possibly influence who you are." Yet clearly not everyone has the reaction described by Grof. As Jansen himself noted, only 30 percent of normal subjects given ketamine thought the events had really happened. The majority, in other words, recognized it as a dream or hallucination rather than an experience that transcended death. In fact, even though Jansen did not say much about his own experiences on ketamine, or his own NDEs for that matter, his article clearly made the point that he himself viewed the NDE as an analogue of a ketamine-induced hallucination rather than a mystical state that eliminated his fear of death.

The point we wish to stress here is that the psychological meanings construed in a unique way by each individual cannot be reduced to neuroscience explanations. If one wants to learn about music, one does not begin by visiting a piano factory.

Jansen's tendency to miss the subtlety and complexity of human experience was again in evidence in his discussion of the criteria of the NDE. In a critique of our discussion (Gabbard and Twemlow, 1989) of the previously published model advocated by Juan Saavedra-Aguilar and Juan Gómez-Jeria (1989), Jansen stated that we required all features of an NDE, both usual and unusual, to be explainable by a unitive theory. In fact, we claimed exactly the opposite. Saavedra-Aguilar and Gómez-Jeria appeared to be claiming to explain all relevant scientific evidence with their model. We simply pointed out some exceptions, such as NDEs occurring in low stress situations. We agree that near-death experiences are not unitive nor is it necessary to make them unitive. We noted in that discussion, as we do here, that the principles of multiple causation and overdetermination must be taken into account to explain psychological phe- 
nomena. Our point was that the model was too general in its attempt to explain virtually every aspect of the NDE.

Throughout his paper, Jansen had a tendency to state what he believes as though it were true by fiat. For example, he wrote, "As might be expected in a mental state with a neurobiological origin, more mundane accounts also occur, such as children who may 'see' their schoolfellows rather than God and angels." It is not apparent to us why this would follow logically from a state that has a neurobiological origin. From our perspective, psychological issues related to the child's developmental phase would more likely determine the fact that some children see schoolfellows (Gabbard and Twemlow, 1984). Again, in our view, Jansen indulged in an unnecessary and unfortunate oversimplification in light of the highly focused content and thrust of his paper. Certainly, the near-death experience is highly variable from person to person and culture to culture (Gabbard and Twemlow, 1984). For example, the tunnel and the being of light in East Indians are often depicted as the River Ganges and a specific guru. In other words, the content and meaning of an NDE are certainly determined as much by intrapsychic factors and by cultural experience as they are by biology.

Near the end of his paper Jansen succinctly summarized a variety of hypotheses for NDEs and then applied his model of protection against glutamate neurotoxicity to see if that model "fit" with the relevant hypothesis. These applications are sketchy and certainly not convincing. Jansen did draw a useful analogy between neurotoxic and neuroprotective mechanisms in his discussion of temporal lobe epilepsy, but in the discussion of whether endorphins can produce NDElike hallucinogenic phenomena, he criticized evidence that certain beta-endorphins have epileptogenic effects. The main thrust of his argument was that experiments with rats cannot be translated to the human situation. This thinking, however, did not stop him from drawing analogies to dogs, who he said have endorphin release associated with death.

Although we have outlined a handful of criticisms of the model, in closing we wish to reiterate our admiration for the scholarly and original work that Jansen has presented here. It is best viewed as a piece of a larger puzzle rather than the solution to the puzzle itself. Nevertheless, we can congratulate Jansen on his contributions to the field and hope that research will be stimulated by his thoughtful essay. 


\section{References}

Bion, W. R. (1963). Elements of psycho-analysis. London, England: Heinemann.

Gabbard, G. O., and Twemlow, S. W. (1984). With the eyes of the mind: An empirical analysis of out-of-body states. New York, NY: Praeger.

Gabbard, G. O., and Twemlow, S. W. (1989). Comments on "A neurobiological model for near-death experiences." Journal of Near-Death Studies, 7, 261-263.

Gabbard, G. O., and Twemlow, S. W. (1991). Do "near-death experiences" occur only near death?-Revisited. Journal of Near-Death Studies, 10, 41-47.

Saavedra-Aguilar, J. C., and Gomez-Jeria, J. S. (1989). A neurobiological model for near-death experiences. Journal of Near-Death Studies, 7, 205-222.

Searle, J. (1992). The rediscovery of the mind. Cambridge, MA: MIT Press. 